\title{
Tailoring of electron flow current in magnetically insulated transmission lines
}

\author{
J. P. Martin, ${ }^{2,3}$ M.E. Savage, ${ }^{1}$ T. D. Pointon, ${ }^{1}$ and M. A. Gilmore ${ }^{2}$ \\ ${ }^{1}$ Sandia National Laboratories, Albuquerque, New Mexico 87185, USA \\ ${ }^{2}$ University of New Mexico, Albuquerque, New Mexico 87131, USA \\ ${ }^{3}$ National Nuclear Security Administration's Kansas City Plant, Kansas City, Missouri 64141, USA
}

(Received 16 September 2008; published 5 March 2009)

It is desirable to optimize (minimizing both the inductance and electron flow) the magnetically insulated vacuum sections of low impedance pulsed-power drivers. The goal of low inductance is understandable from basic efficiency arguments. The goal of low electron flow results from two observations: (1) flowing electrons generally do not deliver energy to (or even reach) most loads, and thus constitute a loss mechanism; (2) energetic electrons deposited in a small area can cause anode damage and anode plasma formation. Low inductance and low electron flow are competing goals; an optimized system requires a balance of the two. While magnetically insulated systems are generally forgiving, there are times when optimization is crucial. For example, in large pulsed-power drivers used to energize high energy density physics loads, the electron flow as a fraction of total current is small, but that flow often reaches the anode in relatively small regions. If the anode temperature becomes high enough to desorb gas, the resulting plasma initiates a gap closure process that can impact system performance. Magnetic-pressure driven ( $z$ pinches and material equation of state) loads behave like a fixed inductor for much of the drive pulse. It is clear that neither fixed gap nor constant-impedance transmission lines are optimal for driving inductive loads. This work shows a technique for developing the optimal impedance profile for the magnetically insulated section of a high-current driver. Particle-in-cell calculations are used to validate the impedance profiles developed in a radial disk magnetically insulated transmission line geometry. The input parameters are the spacing and location of the minimum gap, the effective load inductance, and the desired electron flow profile. The radial electron flow profiles from these simulations are in good agreement with theoretical predictions when driven at relatively high voltage (i.e., $V \geq$ $2 \mathrm{MV})$.

DOI: 10.1103/PhysRevSTAB.12.030401

PACS numbers: $84.70 .+p$

\section{INTRODUCTION}

The efficient transport of electrical power densities originating from large pulsed-power drivers $\left(1-1000 \mathrm{TW} / \mathrm{m}^{2}\right)$ is a critical requirement in operating high power particle beam drivers and intense radiation sources. Magnetic insulation in the vacuum section of these systems is critical, given that the electric field generated between the conductors of the transmission line will usually result in the spacecharge-limited (SCL) emission of electrons from the line's cathode electrode [1,2]. Although the cathode in a vacuum line will freely emit electrons under such electric stress, the self-magnetic field of the transmission line's current inhibits the electrons from reaching the anode [3-23]. When steady-state operation is attained, insulated electrons are confined to a sheath along the cathode and experience an average $\mathbf{E} \times \mathbf{B}$ drift in the direction of power flow. The understanding of the electron flow within these magnetically insulated transmission lines (MITLs) is fundamental to the successful operation of many pulsed-power systems.

Of particular interest are the high-current pulsers used in high energy density physics (HEDP) experiments. Within their vacuum section, the gap spacing between the anode and cathode of each MITL, A-K gap, is an important design parameter that directly impacts the electrical energy transport efficiency of the system. The MITL is optimized through the balance of two competing constraints: the selfinductance of the line and the current carried by vacuumflowing electrons. Those vacuum-flowing electrons reach the anode near the load in many cases. Typically it is desirable to maintain a low system inductance, by minimizing the self-inductance of the MITLs, in order to provide the most efficient transfer of electrical energy to the load [24]. The self-inductance of a MITL is largely determined by its geometry (with some flux reduction as a result of electron flow current) and is lowered through a reduction in the spacing of the A-K gap. Decreasing this selfinductance, however, generally results in an increase in the current conducted by vacuum electrons. This electron current can lead to plasma desorption when concentrated into small regions of the anode (especially near the load), resulting in a significant elevation in the temperature of the surrounding material [25]. If the temperature of the anode surface rises above $\sim 400^{\circ} \mathrm{C}[26,27]$ gas may be desorbed and ionized by the high electric field, forming an anode plasma. The resulting plasma expansion from the anode and ion deposition to the cathode could adversely affect the coupling of power flow into the load [28-34]. It is, there- 
fore, desirable to limit the electron flow current at the load while maintaining the lowest possible vacuum inductance within these systems.

Magnetic-pressure driven ( $z$ pinches and material equation of state) loads behave like a fixed inductor for much of the drive pulse. Within a MITL driving an inductive load, the spatial distribution of the electron flow current varies as it is influenced by the magnetic and electric fields along the transmission line. Because of inductive voltage drop, the voltage varies along the line. Significant voltage differences occur with relatively low load inductances. For example, if the equivalent MITL inductance equals the load inductance, the voltage varies by a factor of 2 along the MITL from inlet to outlet. Choosing an appropriate vacuum impedance profile (i.e., the spacing of the A-K gap as it varies as a function of distance from the load) consistent with the current and local voltage allows for the MITL's self-inductance to be optimized as the total current in flowing electrons is reduced. One such optimization was done for the $Z$ pulser at Sandia National Laboratories [3538]. In the design of the conical MITLs for that system, Stygar et al. [36] demonstrated a procedure for developing radial impedance profiles which were designed to minimize the vacuum inductance of the system while limiting the electron flow current entering the load. This process assumed that the flowing electrons were incapable of returning to the cathode surface, presumably due to an accumulated loss in the total electron energy [19]. The design was implemented using localized pressure-balance calculations, which permitted the magnitude of the electron flow current to be expressed in terms of the local voltage and total current [12], with the additional restriction that electron flow current could never decrease in the direction of power flow. The geometry of the vacuum section was optimized through iterative computer modeling in order to produce the desired electrical performance at the load [37]. Clearly, when vacuum-flowing electrons are sufficiently insulated from reaching the anode and cannot return to the cathode, the least inductive A-K gap profile for this geometry results from a radially invariant electron flow distribution.

Particle-in-cell (PIC) calculations performed on the vacuum section of the $Z$ pulser $[39,40]$ have demonstrated that the local electron flow current operates at values which are closely approximated by the local application of the 1D pressure-balance model developed in Ref. [15], where the vacuum impedance is varying slowly with radius. Furthermore, these PIC simulations show that the electron flow current near the load is considerably less than what was originally predicted from circuit model calculations [37]. It is hypothesized in Ref. [39] that this is a result of electrons being retrapped (returned to the cathode) as the local vacuum impedance gradually increases toward the load. Well-diagnosed studies on a radiographic x-ray driver (which has higher fractional electron flow than $Z$ ) have measured the effects of electrons returning to the cathode in some cases [41,42]. A design approach that accounts for the possibility of electrons being returned to the cathode could potentially lead to A-K gap spacing profiles which are successful in further reducing the electron flow current near the load of these MITLs. Decreasing the current in vacuum-flowing electrons near the load would reduce the amount of electron heating of the anode, and improve power flow efficiency of large pulsed-power drivers [43].

In an effort to derive analytically the optimal A-K gap profile for a radial MITL driving a $z$-pinch load, Savage [44] developed an expression for a constant electron flow impedance profile with regards to the line's spatially varying voltage and vacuum impedance. This distributed impedance was derived under the assumption that the system possessed electromagnetic transit times that were shorter than the current rise time; this led to a profile which was formulated in terms of the size and location of the MITL's minimum gap, and the inductance contained within the radius of the minimum gap. Savage et al. [45] later generalized these calculations to allow for a spatially varying electron flow. Those calculations are shown in Sec. II A below, and are equally valid whether any excess flowing electrons reach the anode or the cathode. In that model, impedance profile solutions were generated numerically (using a conventional root-solving algorithm), which corresponded to a prescribed rate of fractional electron current change in the direction of power flow. Later work introduced the general analytic mathematical solution to the profile calculations. These methods neglect the expansion of electrode plasmas across the A-K gap (i.e., gap closure) and assume that the load inductance does not change as a function of time. Typical (and efficient [24]) HEDP load designs behave as essentially constant inductance during much of the rise of the line current allowing them to be modeled as an inductive cavity throughout the initial part of the drive pulse [46,47]; by design their inductance is essentially constant early in the drive pulse.

In this article we develop a design methodology centered on the geometrical encoding of impedance profiles into the A-K gaps of strongly insulated MITLs feeding inductive loads. When reducing the electron flow current at the load, these impedance profiles optimize the MITL's selfinductance while attempting to maintain an adiabatic change in the spatial distribution of the electron flow along the line. Although this method can be generalized to encompass any vacuum line geometry, the derivation of the impedance profiles given in Sec. II is restricted to geometries that are generated from $2 \mathrm{D}$ cross sections possessing either translational or rotational symmetry (orthogonal to the vacuum electron drift). Furthermore, while the remainder of this article focuses on purely inductive systems, as a simplifying assumption, any system with transit times shorter than the drive voltage rise time, and a load much lower in impedance than the MITL vacuum impedance, 
would behave similarly. The model presented in Sec. II A assumes that the line voltage is well above the rest mass of an electron, i.e. $V \cong 511 \mathrm{kV}$, and that the vacuum electron flow is a function of the MITL's local voltage and local wave impedance. Analytical calculations which utilize the localized 1D pressure balance of the electron sheath are used to provide a lossless first-order approximation for the relative behavior of the electron flow current as it varies as a function of distance from the load. Gap profiles are derived in Sec. II B and are represented by functions that vary slowly with radius (for a radial disk MITL), are monotonically decreasing in the direction of power flow, and are uniquely defined by the desired spatial distribution of the electron current. For numerical particle simulation reasons, the design incorporates a flat cathode with a curved anode into the MITL design. A flat cathode avoids numerical issues associated with "stair-stepping" perturbations in the high-resolution PIC calculations. Those calculations will be used to validate the model in Sec. III.

While the primary focus of this article is on improving the electrical efficiency of a high-current driver (optimizing vacuum inductance in tandem with reducing the electron flow current near the load), it should be noted that several additional challenges exist for coupling power flow into the load. Reductions in the load current (due to losses in the vacuum section) are typically due to effective gap closure within the MITL, resulting from anode and cathode plasma expansion [48]. In this article, we are effectively assuming that the minimum gap spacing is large in comparison to the product of the plasma's expansion velocity and the time scale of the pulse. The main concern, as discussed in Sec. III, is the impedance profile's effect on the bunching of the electron flow near the load as a result of the deceleration of the vacuum electron drift in the direction of increasing localized impedance [22]. There exists a limitation as to what extent the electron flow rate can diminish (in the direction of power flow) before the subsequent accumulation of excess space-charge counteracts the retrapping of vacuum electrons to the cathode [39]. Furthermore, if a sufficiently large linear current density is present near the load, resistive (i.e., Ohmic) heating of the anode surface will result in the presence of ion space charge $[33,34]$ which will also limit the reduction of the electron flow current [30].

\section{ANALYTICAL RETRAPPING MODEL}

The calculations below demonstrate a general method for estimating the A-K gap profile for a strongly insulated MITL when provided common parameters. PIC simulations of previous high-current drivers suggest that the electron flow current within these inductive systems tends to the local equilibrated theoretical value $[39,40]$. It is therefore assumed, a posteriori, that the magnitude of the electron flow, at a certain distance from the load, can be determined analytically via the local quasistatic balance between the magnetic field pressure and electric tension acting on the vacuum electron sheath. This would imply that the impedance profile of the line, and thus the MITL's self-inductance as a function of distance from the load, sets the relative spatial behavior of the electron flow's magnitude. This tailoring of the electron flow provides a decrease in the magnitude of the electron flow current in the direction of power flow when vacuum-flowing electrons are capable of either being returned to the cathode, or reaching the anode. Electron flow to the cathode (retrapping) is much more energetically desirable in practice because electrons reach the cathode with substantially less kinetic energy than electrons striking the anode. Because of the magnetic field, electrons can generally only reach the anode by aggregating into vortices with large space-charge fields. Those electrons impact the anode with kinetic energy equal to the MITL voltage at that point (typically $\mathrm{MeV}$ ). In fact, the high-resolution simulations in Sec. III show that for tailored electron flow e-folding distances of one meter or more, electrons stay close to the cathode, and return to the cathode during the current rise.

The exact distribution of the MITL's electron flow current is dependent on large scale variations within the spatial distribution of the vacuum electrons' total energy. Electrons that continually gain total energy will be returned to the cathode surface [49]. This is possible for an orbiting electron, within the MITL's A-K gap, when it is extracting energy from the time-varying magnetic field. During the rise of the current pulse, electrons drifting along the cathode experience an increase in the magnetic flux enclosed by their orbits. The electromotive force associated with this increasing flux performs work on the electrons to increase their total kinetic energy through betatron acceleration [50,51]. The energy typically gained within the PIC calculations presented in Sec. III is on the order of $750 \mathrm{eV} / \mathrm{cm}$ for an electron with an average height of $1 \mathrm{~mm}$ (above the cathode), at a radius of $0.2 \mathrm{~m}$ (from the load) and $7.5 \times 10^{13} \mathrm{~A} / \mathrm{s}$ current slew rate. If the energy gained through this process is higher than the rate of electron energy loss, a mechanism is provided which is capable of returning vacuum-flowing electrons to the cathode conductor.

Drifting electrons can lose energy through synchrotron radiation [52,53] and effective collisions through electron flow instabilities resulting from electrostatic- and electromagnetic-field fluctuations [54]. The energy radiated from MITL electrons, gyrating within the vacuum sheath, constitutes a negligible fraction of the electron's total energy within the nanosecond time scales in which these drivers typically operate and will therefore be omitted from the proposed model. A decrease in the total electron energy can affect the electron flow when vacuum-flowing electrons undergo effective collisions, broadening the electron sheath. A comparison of a proposed collisional model and collisionless predictions 
against a wide range of pulsed power experiments suggest that effective collisions might produce non-negligible effects on the electrical performance of physically relevant MITLs [54]. These effects are not evident in the highresolution two-dimensional radial disk PIC simulations described in Sec. III and are not included in the simple model presented in Sec. II A, which is derived through only pressure-balance considerations. Likewise, the PIC calculations, discussed in Sec. III, provide a self-consistent model of the magnetic induction within the vacuum line but do not include models for electron energy loss due to radiation, neutral particle interactions, or classical charged particle collisions. Therefore, the model developed in Sec. II A will provide an initial design for MITL impedance profiles albeit a complete description would require the inclusion of electron energy loss considerations.

\section{A. Impedance profiles}

Consider the conceptual design, as shown in Fig. 1, for an inductively loaded MITL utilizing a spatially varying A-K gap profile, which is tapered from the power inlet to the load. It is assumed here that the geometry is cylindrically symmetric, either defining a radial disk or a tapered coaxial line depending upon the axis of revolution. The inductance of the load is assumed to be fixed. The following calculations, in reference to Fig. 1, define the enclosed inductance as a function of distance (from the load) and the relative change in the magnitude of the electron flow current in the direction of power flow. Assuming that the line total current is azimuthally uniform, the impedance

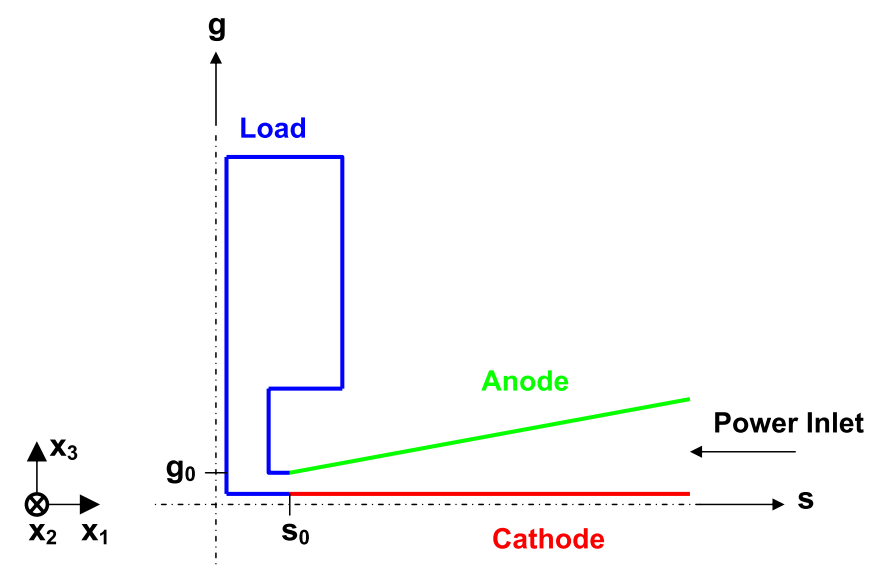

FIG. 1. (Color) Inductively loaded MITL with spatially varying A-K gap profile. This configuration is cylindrically symmetric about either the $\hat{g}$ or $\hat{s}$ axis depending upon the intended geometry. A radial disk configuration would be generated by revolving the $\hat{s}$ axis about the $\hat{g}$ axis such that $\left(x_{1}, x_{2}, x_{3}\right) \rightarrow$ $(r, \theta, z)$ for a cylindrical curvilinear coordinate system. For a coaxial line, the $\hat{g}$ axis is revolved about the $\hat{s}$ axis such that $\left(x_{1}, x_{2}, x_{3}\right) \rightarrow(z,-\theta, r)$; the gap spacing in this case is defined as the difference between the radius at the edge of the anode and the radius at the edge of the cathode. profiles are derived from the local vacuum wave impedance of the transmission line and the 1D pressure balance of the electron sheath in terms of current and local voltage.

We begin by defining the enclosed inductance at any position along the line, $s$, as the summation of the inductance of the load, $L_{0}$, and the integration of the transmission line's inductance per unit length,

$$
L(s)=L_{0}+\frac{1}{c} \int_{s_{0}}^{s} Z_{v}\left(s^{\prime}\right) d s^{\prime},
$$

where $s_{0}$ is the location of the load cavity, $s$ is the dimension of power flow (i.e., $s \rightarrow r$ for a radial disk geometry and $s \rightarrow z$ for a coaxial line), and the inductance per unit length is equal to the wave impedance, $Z_{v}$, divided by the wave propagation velocity in vacuum, $c$. The spatial distribution of the MITL's anode potential is directly related to this inductance profile. We assume that at each position, the anode and cathode currents can be expressed in terms of the line voltage, $V_{a}$, through local electromagnetic pressure-balance considerations [15,21],

$$
V_{a}=Z_{v} \sqrt{I_{a}^{2}-I_{c}^{2}}-\frac{m_{e} c^{2}}{2 e}\left(\frac{I_{a}^{2}}{I_{c}^{2}}-1\right)
$$

where $I_{a}$ and $I_{c}$ are the line currents conducted through the anode and cathode, respectively, $m_{e}$ is the electron rest mass, and $e$ is the fundamental electron charge. The total current flowing in the anode is divided in the return path between the current flowing in the cathode and the current flowing through the vacuum electron flow, i.e. $I_{e}=I_{a}-$ $I_{c}$. As a result, the local electron flow current is implicitly contained within the analytical formulation given in (2). If it is assumed that the electron flow is strongly insulated; that is, the anode current is much greater than the current carried by vacuum-flowing electrons, then a relation for the electron flow current can be extracted from Eq. (2). The electron current is well approximated by the explicit equation

$$
I_{e} \approx \frac{V_{a}^{2}}{2 I_{a} Z_{v}^{2}} .
$$

Systems in which the electron flow is a small fraction of the total current (and therefore have electron flow close to the cathode) have an inductance profile close to the vacuum inductance profile. Assuming the electrons are flowing parallel to the electrodes, the inductance per unit length of the transmission line is due to the spatial derivative (in the power flow direction) of the total magnetic flux across the A-K gap. The total flux is the sum of the flux between the anode and the electron sheath, and the flux between the electron sheath and the cathode. We define the total inductance of the line as the ratio of magnetic flux to the anode current,

$$
L=\frac{1}{I_{a}}\left[\Phi_{a}+\Phi_{c}\right]
$$


where $\Phi_{a}$ is the magnetic flux on the anode side of the electron sheath and $\Phi_{c}$ is the magnetic flux on the cathode side of the electron sheath. The spatial derivative of this inductance gives the inductance per unit length of the transmission line,

$$
L^{\prime}=\frac{1}{I_{a} c}\left[I_{a} Z_{f}+I_{c}\left(Z_{v}-Z_{f}\right)\right]=\frac{1}{c}\left[Z_{f}+\frac{I_{c}}{I_{a}}\left(Z_{v}-Z_{f}\right)\right],
$$

where $Z_{f}$, the flow impedance [21], is the effective wave impedance associated with the distance between the anode and the location of the electron sheath (or the centroid of the sheath if the sheath has non-negligible thickness). If the electron sheath is close to the cathode, the flow impedance approaches the vacuum impedance. For the PIC simulations presented in Sec. III, the average flow impedance was calculated to be roughly 0.95 of the vacuum impedance, and the cathode current is 0.9 of the anode current. The inductance per unit length calculated by Eq. (5) with those values is thus 0.995 of the vacuum value. It is therefore reasonable, in this case, to neglect the flux reduction due to the electron flow current. This will be used as a simplifying assumption when deriving the impedance profiles below. It is commonly assumed that the effective inductance per unit length is related solely to the flow impedance in a MITL by

$$
L^{\prime}=\frac{Z_{f}}{c}
$$

however, Eq. (6) is generally incorrect and substantially underestimates the inductance when the electron flow is a small fraction of the total current.

The expression for the electron flow current can be related to the MITL's self-inductance through a substitution for the line voltage, which is equivalent to the enclosed inductance, as a function of position, scaled by the time derivative of the anode current,

$$
I_{e}(s) \approx \frac{L(s)^{2}\left(\dot{I}_{a}\right)^{2}}{2 I_{a} Z_{v}(s)^{2}}=\frac{\left(\dot{I}_{a}\right)^{2}}{2 c^{2} I_{a}}\left(\frac{L}{L^{\prime}}\right)^{2},
$$

where the vacuum wave impedance, $Z_{v}$, has been expressed in terms of the enclosed inductance given in Eq. (1). Here the diacritic marks above $I_{a}$ and $L$ denote time derivatives and spatial derivatives, respectively. Assuming that the electromagnetic transit time of the system is short in comparison to the rise time of the current pulse, i.e. $d I_{a} / d s \approx 0$, the change in the electron flow current along the line is then given by

$$
\frac{\partial I_{e}}{\partial s}=\frac{\left(\dot{I}_{a}\right)^{2}}{c^{2} I_{a}}\left[\frac{L}{L^{\prime}}-\frac{L^{2} L^{\prime \prime}}{\left(L^{\prime}\right)^{3}}\right]
$$

Dividing Eq. (8) by the total electron flow current yields the fractional change in the electron flow current with respect to position, which is defined as the retrapping rate,

$$
\beta=\frac{1}{I_{e}} \frac{\partial I_{e}}{\partial s}=2 \frac{\left(L^{\prime}\right)^{2}-L^{\prime \prime} L}{L L^{\prime}} .
$$

This is the fractional rate (per unit distance) at which the electron flow current is changed in the direction of the power flow. While this parameter could, in general, be a function of position, it is defined here to be spatially invariant to maintain an adiabatic change in the electron flow. For configurations that have reducing electron flow, the maximum adiabatic reduction in electron current occurs with the highest stable constant flow reduction over the longest possible distance. Equation (9) is a nonlinear differential equation that describes the inductance of the transmission line as a function of both position and the retrapping rate of the vacuum electrons to the cathode. The closed form solution of Eq. (9), assuming a constant $\beta$, is readily obtained through an exponential substitution, which results in the following inductance profile:

$$
L(s, \beta)=C_{2}(\beta) \exp \left\{-\frac{2 C_{1}}{\beta} \exp \left[-\frac{\beta}{2}\left(s-s_{0}\right)\right]\right\},
$$

where

$$
\begin{gathered}
C_{1}=\frac{Z_{v}\left(s_{0}\right)}{L_{0} c}, \\
C_{2}(\beta)=L_{0} \exp \left[\frac{2 Z_{v}\left(s_{0}\right)}{c L_{0} \beta}\right] .
\end{gathered}
$$

Although this expression possesses a singular point at $\beta=$ 0 , the inductive profile for a constant reducing flow rate exists as a special solution whose asymptotic behavior is not accessible from the general solution. This solution can be obtained either by substituting $\beta=0$ directly into Eq. (9) and solving the resulting ordinary differential equation or by taking the limit of Eq. (10) as $\beta$ approaches zero,

$$
\lim _{\beta \rightarrow 0} L(s, \beta)=L_{0} \exp \left[\frac{Z_{v}\left(s_{0}\right)\left(s-s_{0}\right)}{L_{0} c}\right] .
$$

Equations (10)-(13) thus constitute the complete solution of Eq. (9), and provide a unique inductive profile for a chosen retrapping rate. The vacuum inductance at any distance from the load is therefore influenced by the chosen retrapping rate which in turn sets the fractional change in the magnitude of the electron flow per unit length, as it varies with position. The vacuum impedance of the line appears within the constants of integration and depends upon the geometry of the A-K gap. This relationship allows for the gap spacing, as a function of position, to be expressed directly in terms of the retrapping rate; this will be demonstrated for a radial disk configuration below. 


\section{Constant electron flow}

If the vacuum electrons are inhibited from reaching either the anode or the cathode, then the lowest inductance case corresponds to a uniform electron flow distribution in the power flow direction. This is the case of constant electron flow, or a zero retrapping rate. The inductive profile for a zero retrapping rate, $\beta=0$, is given by Eq. (13). The vacuum impedance in the numerator of the exponential function's argument depends upon the geometry of the MITL. For a radial disk transmission line, the local wave impedance is given by

$$
Z_{v}(r)=\frac{1}{2 \pi} \sqrt{\frac{\mu_{0}}{\varepsilon_{0}}} \frac{g}{r}
$$

where $g$ is the distance across the A-K gap at radius $r$. The impedance profile corresponding to a constant electron flow within this geometry, denoted by $Z_{v 0}(r)$, can be expressed as a function of radius, solely in terms of the transmission line's geometrical constants and the inductance of the load, by substituting Eq. (13) into Eq. (1) and setting $s \rightarrow r$,

$$
Z_{v 0}(r)=c \frac{\partial L}{\partial r}=\frac{\eta_{0}}{2 \pi} \frac{g_{0}}{r_{0}} \exp \left[\frac{\eta_{0}}{2 \pi} \frac{g_{0}\left(r-r_{0}\right)}{L_{0} r_{0} c}\right],
$$

where the term $Z_{v}\left(s_{0}\right)$ in Eq. (13) has been replaced with Eq. (14), evaluated at $r=r_{0}$, and $\eta_{0}=\sqrt{\mu_{0} / \varepsilon_{0}}$.

\section{Reducing electron flow}

For a positive retrapping rate, the analytical model predicts a decreasing electron flow current in the direction of power flow. This can be regarded as the ideal case; electrons experience an increase in their total energy as they drift through an increasing magnetic field exclusive of energy loss considerations. Larger retrapping rates correspond to a greater reduction of the electron flow current near the load, and that current is generally returned to the cathode conductor during the current rise. Realistically, however, the degree to which the electron flow can be returned to the cathode will be limited by the accumulation of excess space charge within this region [22,39].

The impedance profile resulting from a nonzero retrapping rate must be derived from the general solution given in Eq. (10). As an example, assume that there is an appreciable change in the electron flow versus position such that the amplitude of the current is attenuated by a factor of $1 / e$ for every meter traversed in the direction of power flow, i.e. $\beta=1 \mathrm{~m}^{-1}$. The constants of integration, in a radial geometry, simplify to

$$
\begin{gathered}
C_{1}=\frac{Z_{v}\left(r_{0}\right)}{L_{0} c}, \\
C_{2}=L_{0} \exp \left[\frac{2 Z_{v}\left(r_{0}\right)}{L_{0} c}\right],
\end{gathered}
$$

where the local wave impedance, $Z_{v}\left(r_{0}\right)$, is given by Eq. (14). Using these constants of integration, the vacuum wave impedance for the $\beta=1 \mathrm{~m}^{-1}$ reducing flow profile, $Z_{v 1}(r)$, is given by the following expression:

$$
\begin{aligned}
Z_{v 1}(r)= & \frac{\eta_{0}}{2 \pi} \frac{g_{0}}{r_{0}} \exp \left\{\frac{\eta_{0}}{\pi} \frac{g_{0}}{c L_{0} r_{0}}-\frac{\eta_{0}}{\pi} \frac{g_{0}}{c L_{0} r_{0}}\right. \\
& \left.\times \exp \left[-\frac{1}{2}\left(r-r_{0}\right)\right]-\frac{1}{2}\left(r-r_{0}\right)\right\} .
\end{aligned}
$$

\section{B. A-K gap profiles}

In order to design the geometrical profile for a particular flow rate, the gap spacing versus radius must be extracted from either Eq. (15), for constant electron flow, or Eq. (18), for the $\beta=1 \mathrm{~m}^{-1}$ radially reducing flow. Substituting either of these expressions into Eq. (14) eliminates the characteristic impedance term and defines the gap spacing by a unique one-dimensional function possessing three parameters: the radius of the minimum gap spacing, $r=$ $r_{0}$, the minimum A-K gap spacing, $g=g_{0}$, and the inductance, $L_{0}$, of the cavity inside the minimum gap. When these parameters are fixed, the gap profile (and maximum gap spacing located at the outer perimeter of the disk) will vary for each retrapping rate. If it were desired to set the location and size of the maximum gap, the minimum gap spacing would become a function of the retrapping rate.

The gap profiles for two separate retrapping rates are given in Fig. 2. In each of these cases, the maximum gap spacing is constrained $(32.6 \mathrm{~mm}$ at a radius of $46 \mathrm{~cm}$ ). The minimum gap spacing is shown to grow larger with an

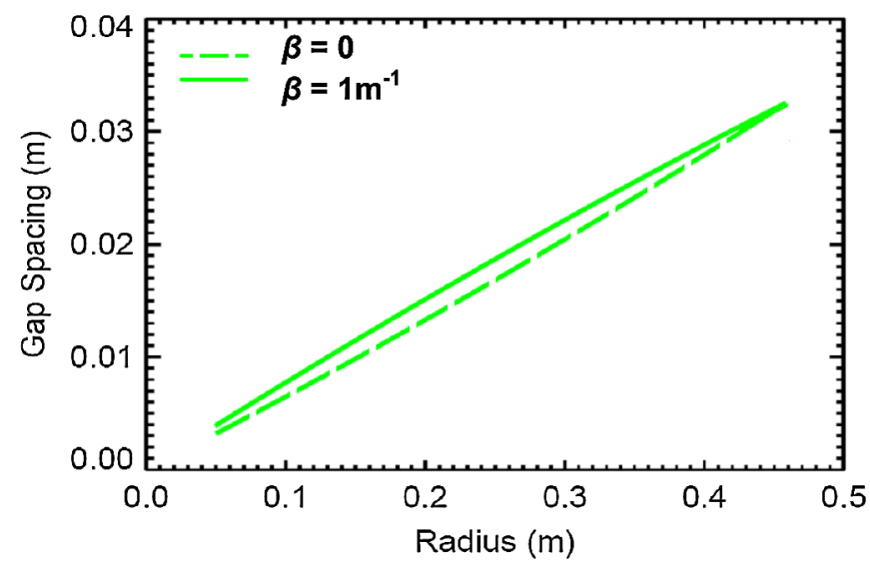

FIG. 2. (Color) The A-K gap spacing as a function of radius for a radial disk MITL, plotted for $0.05 \mathrm{~m} \leq r \leq 0.46 \mathrm{~m}$. The inductance of the load is $51.2 \mathrm{nH}$; the maximum gap spacing is $32.6 \mathrm{~mm}$ and is located at the edge of the anode disk $(r=$ $0.46 \mathrm{~m}$ ). The bottom curve represents the curvature of the anode which corresponds to a radially constant electron flow profile. The concave curvature of the anode becomes more pronounced for larger retrapping rates. The $\beta=1 \mathrm{~m}^{-1}$ curve represents an anode profile which results in $1 / e$ scaling of the electron flow current for every meter towards the load. 


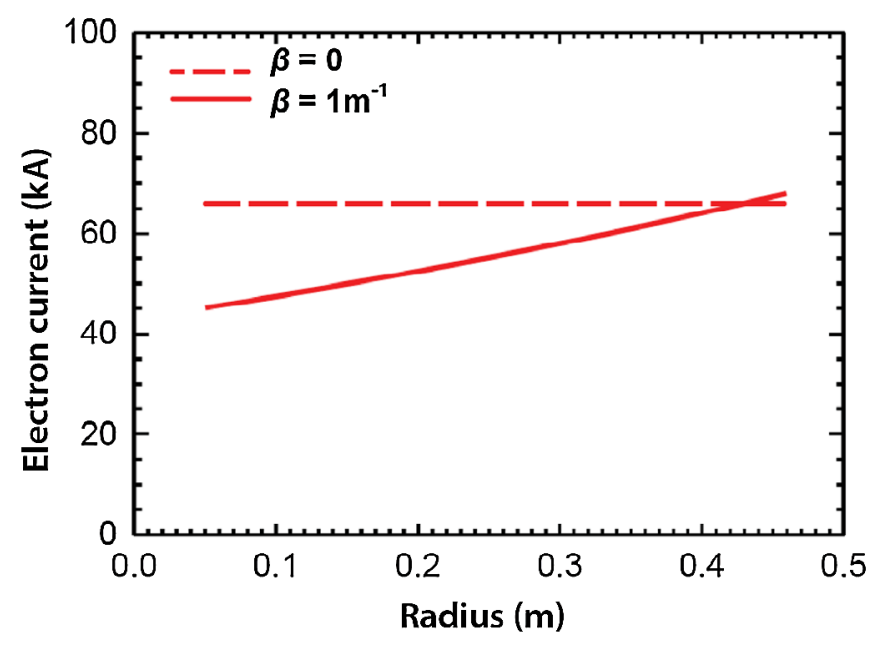

FIG. 3. (Color) Magnitude of the analytically calculated electron flow current as a function of radius for a radial disk MITL, plotted for $0.05 \mathrm{~m} \leq r \leq 0.46 \mathrm{~m}$. The inductance of the load is $51.2 \mathrm{nH}$; the maximum gap spacing is $32.6 \mathrm{~mm}$ and is located at the edge of the anode disk $(r=0.46 \mathrm{~m})$. The upper curve represents the constant electron flow profile resulting from an insulated line where electrons are not returned to the cathode surface. The lower curve represents a positive retrapping rate where the magnitude of the current is attenuated by a factor of $1 / e$ with every meter towards the load from the power inlet. The total anode current at this time is approximately 1.5 MA.

increase in $\beta$. Figure 3 illustrates the calculated electron flow given some typical parameters. In this particular example the forward voltage wave is $5 \mathrm{MV}$ peak with a total line current that rises to $\cong 300 \mathrm{kA}$ within a $20 \mathrm{~ns}$ interval. The inductance of the load cavity is set to $51.2 \mathrm{nH}$

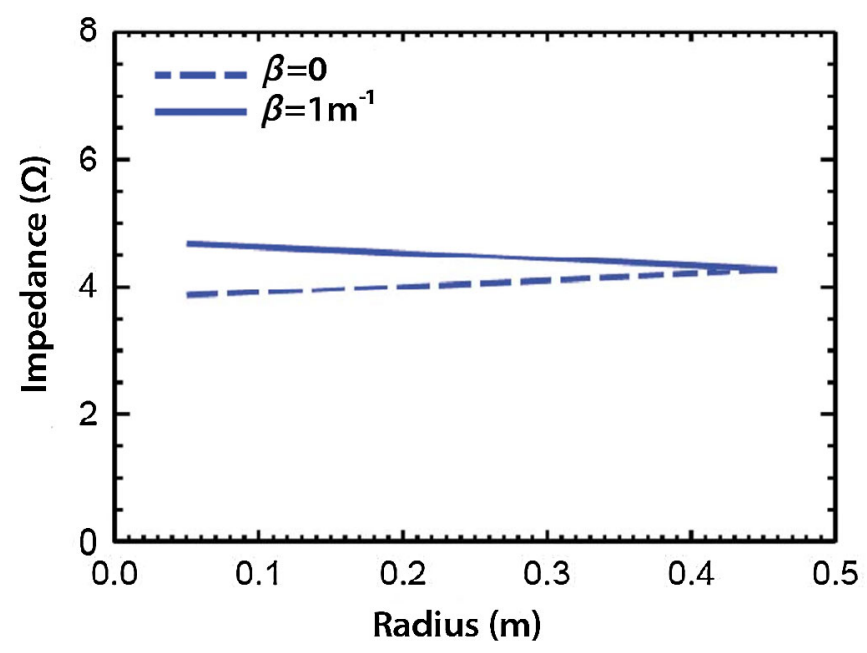

FIG. 4. (Color) The vacuum impedance as a function of radius for a radial disk MITL, plotted for $0.05 \mathrm{~m} \leq r \leq 0.46 \mathrm{~m}$. The inductance of the load is $51.2 \mathrm{nH}$; the maximum gap spacing is $32.6 \mathrm{~mm}$ and is located at the edge of the anode disk $(r=$ $0.46 \mathrm{~m}$ ). For a strongly insulated transmission line it would be expected that this impedance profile is close to the operational impedance of the MITL. for each of the profiles. As the retrapping rate increases, the relative radial decrease in the magnitude of the electron flow toward the load becomes more dramatic. The impedance profiles for constant and reducing flow are shown in Fig. 4. These impedance profiles illustrate that a decrease in the electron flow current at the load comes at the cost of increasing the self-inductance of the MITL. In this particular case, decreasing the electron flow current by $32 \%$ at $r=0.05 \mathrm{~m}$ (Fig. 3) corresponds to an increase of $10 \%$ in MITL inductance (Fig. 4). For reference, decreasing the electron flow $32 \%$ with a constant fractional MITL impedance increase would require a $21 \%$ impedance (and therefore MITL inductance) increase. In the PIC simulation, covered in Sec. III, the average flow impedance over the radial span of the MITL was calculated to be roughly 0.9 of the vacuum impedance. Because of the small electron flow and the high flow impedance, the vacuum inductance accurately determines the voltage, meeting the assumptions of the model.

\section{PARTICLE-IN-CELL SIMULATIONS}

The simulations supporting this work were performed using the finite difference-time domain (FDTD), 3D electromagnetic, PIC code QUICKSILVER [55]. This code utilizes standard FDTD field-solvers and electromagnetic "particle-pusher" PIC algorithms [56] to resolve the time-dependent electrodynamics within the disk MITL. The magnetic induction within the line is modeled selfconsistently and influences the electron trajectories within the A-K gap. QUICKSILVER does not possess models for radiation, neutral particle physics, or classical charged particle collisions and therefore cannot account for electron energy lost as a result of these mechanisms; this is consistent with the assumptions made in the development of the simple 1D analytical model outlined in Sec. II A.

The conductor geometries for a typical radial disk MITL simulation are shown in Fig. 1, where $\left(x_{1}, x_{2}, x_{3}\right) \rightarrow$ $(r, \theta, z)$. The curvature of the anode depends upon the particular impedance profile under study. The conductors are assumed to be cylindrically symmetric and extend outward, with increasing radius, from the load to the inlet of the transmission line feed. Previous PIC modeling of similar geometries have demonstrated that the electron flow is in effect azimuthally uniform [31]. Thus, 2D PIC simulations were conducted in the $\hat{r}-\hat{z}$ plane and the total electron current was calculated assuming cylindrical symmetry in the $\hat{\theta}$ direction. This allowed for greater computational resolution while maintaining moderate time intervals in which to conduct the simulations. To study the effects of fielding actual current monitors ( $B$-dot loops) within this design, concentric annular grooves are included (for housing the diagnostics) and are evenly distributed, radially, along both the anode and cathode of the simulation. Each groove consists of an extruded rectangular channel within the conductor plate of a depth of $1 \mathrm{~mm}$ 
and is characterized by an inner radius, $r_{i}$, and outer radius, $r_{o}$, where $r_{o}-r_{i}=1 \mathrm{~mm}$ is the $2 \mathrm{D}$ radial length of the groove. The azimuthal symmetry of the grooves allows them to be included in the 2D simulation and the rectangular cross section avoids introducing stair-stepping perturbations within the electron sheath region. Initial calculations and simulations (with and without the grooves) revealed that the presence of these grooves was negligible because of the short (subnanosecond) time scale required for the grooves to fill with electrons.

Within the strongly insulated systems considered in this article, particularly late in the pulse, the thickness of the electron sheath near peak current is thin compared to the A-K gap, as a result of the small ratio of the electric to magnetic fields. Consequently, the simulation region along the cathode conductor boundary, where the electron emission is initiated, is of particular importance as a result of its relatively large simulated particle density. In order to resolve accurately the electron trajectories close to the cathode, it is critical to utilize a sufficient number of simulation cells. For the simulations described below, the electron sheath thickness is approximately $1.2 \mathrm{~mm}$ late in the pulse. Several simulations were conducted (varying the cell density in each case) to determine an appropriate gridding for this region. A portion of these simulations were performed into a resistive load such that the electron flow could be equilibrated. These static simulations offer an advantage over the use of an inductive load in that it aids in the isolation of numerical problems within the simulation, improving the resolution of some effects. It was determined that cell sizes with an axial length of $200 \mu \mathrm{m}$ (corresponding to six cells across the electron sheath) provided adequate resolution across the electron sheath. The remainder of the A-K gap (edge of the electron sheath to the anode) utilized larger cells with a maximum axial length of $428 \mu \mathrm{m}$ (adjacent to the anode). The radial cell sizes ranged from $740 \mu \mathrm{m}$ to $1.07 \mathrm{~mm}$; the smallest of these were positioned over the current monitor grooves along the cathode.

The initial boundary condition across the transmission line inlet was generated from an experimental forward voltage wave that supplied a potential of roughly $500 \mathrm{kV}$ over a pulse width of $50 \mathrm{~ns}$. The analytical model presented in this article was developed under the assumption that the sheath thickness is thin compared with the distance across the MITL A-K gap. Therefore, the amplitude of the simulated driver pulse was numerically increased by an order of magnitude, $V=5 \mathrm{MV}$, reducing the effect of the electron space-charge potential [12]. The load cavity for these simulations possessed a load inductance, $L_{0}=51.2 \mathrm{nH}$, and was located just inside the radius of the minimum gap, in conjunction with the initial design used in each of the impedance profiles. The simulated particles, which constitute the electron sheath current, were initiated via emission cells, located along the cathode surface, and modeled using a standard SCL emission algorithm [57]. Initially these cells are nonemitting but are activated when the electric field normal to the conductor boundary exceeds a predetermined threshold value, which was set to $200 \mathrm{kV} / \mathrm{cm}$. In the high-voltage simulations presented here, the average electric field quickly exceeds commonly used thresholds for bare metal surfaces [58] and so the value of the emission threshold is not critical.

Two-dimensional PIC calculations were conducted for a constant flow profile, $\beta=0$, a reducing flow profile, $\beta=$ $1 \mathrm{~m}^{-1}$, and a substantially decreasing electron flow profile, $\beta=3.7 \mathrm{~m}^{-1}$. This wide range in retrapping rates, $\beta$, was necessary to construct a comprehensive picture of the radial electron flow near different operating regimes. Inlet voltage and current from a high-voltage simulation is shown in Fig. 5. The radial flow profiles generated by these simulations are compared with model predictions in Fig. 6. The simulated electron flow current was determined from the anode and cathode currents, i.e. $I_{a}-I_{c}$, which were calculated using magnetic field measurements along the surface of the electrodes at several discrete radial locations. The theoretical model developed in Sec. II predicts that the electron flow current for any given retrapping parameter, $\beta$, will be of the form:

$$
I_{e}(r)=I_{e: r_{\max }} \exp \left[\beta\left(r-r_{\max }\right)\right],
$$

where $I_{e: r_{\max }}$ is the maximum electron flow current for a reducing flow profile and $r_{\max }$ is the maximum radial length of the disk MITL. The simulation values corroborate

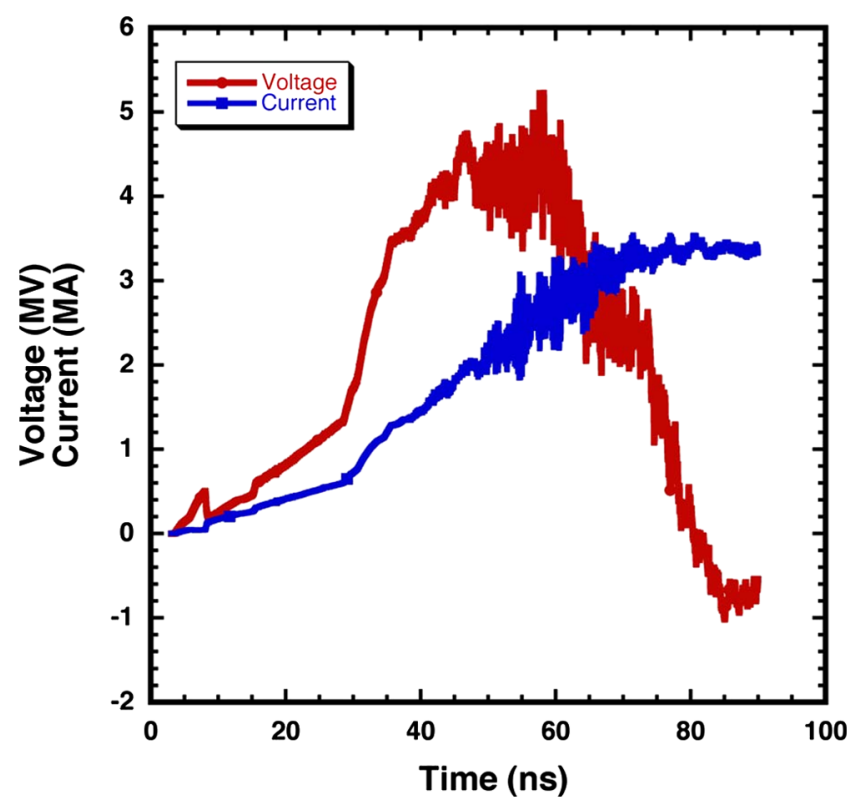

FIG. 5. (Color) Inlet voltage and current for the high-voltage simulation. The oscillations starting at $50 \mathrm{~ns}$ are due to electron instability as electron drift velocity slows due to stable or falling voltage and increasing currents. The vacuum-flowing electron current at $55 \mathrm{~ns}$ is $65 \mathrm{kA}$, or $4.3 \%$ of the total current at that time. 


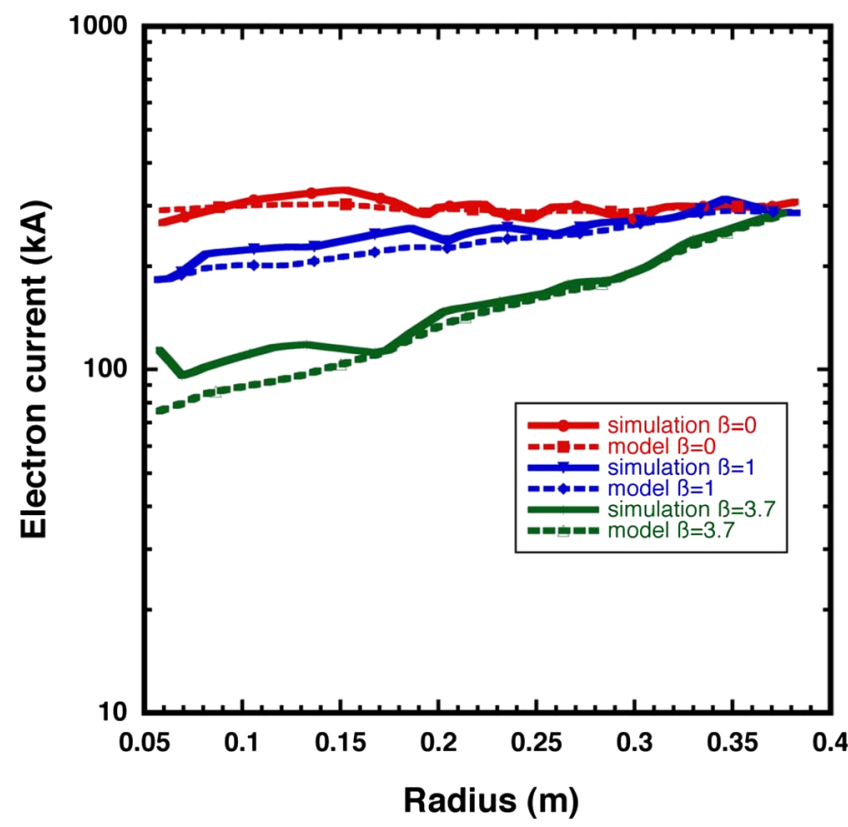

FIG. 6. (Color) Simulation electron flow for the $\beta=0,1$, and 3.7 per meter radial profiles at high voltage (i.e., $V=5 \mathrm{MV}$ ). $\beta=0$ represents constant electron flow. $\beta=1 \mathrm{~m}^{-1}$ represents a reduction of electron flow which decreases as $1 / e$ for every meter towards the load. $\beta=3.7 \mathrm{~m}^{-1}$ represents the highest retrapping rate evaluated.

the theoretical predictions to a great extent. The change in retrapping rates partitions Fig. 6 into three distinct sections. The upper curve represents the constant electron flow design, $\beta=0$, while the middle $\beta=1 \mathrm{~m}^{-1}$ curve is shown to decrease the relative flow current to $1 / e(37 \%)$ for every meter in the power flow direction. The lower $\beta=$ $3.7 \mathrm{~m}^{-1}$ curve represents an aggressive retrapping rate that is characterized by a relative change in electron flow of $1 / e$ for every $27 \mathrm{~cm}$ in the power flow direction. The separation between the curves illustrates the relative decrease in electron flow at inner radius for higher retrapping rates. The simulated electron flow curves vary somewhat from the model prediction because of temporal fluctuations in the simulation electron flow current.

A simulation result showing the simulated retrapping rate for the constant-impedance profile is shown in Fig. 7. This curve predicts an approximately zero retrapping rate (as designed) within the time interval of 42 to $54 \mathrm{~ns}$; this corresponds to times when voltage was high enough to cause electron emission, and electron flow was predominately parallel to the cathode surface, satisfying the assumptions of the analytical model. The largely temporally independent retrapping rate validates one important model assumption: while the magnitude of the electron current varies greatly, the fractional electron flow is largely constant with the so-tailored profile.

Figure 6 demonstrates the ability to tailor the electron flow current through a predetermined impedance profile, as

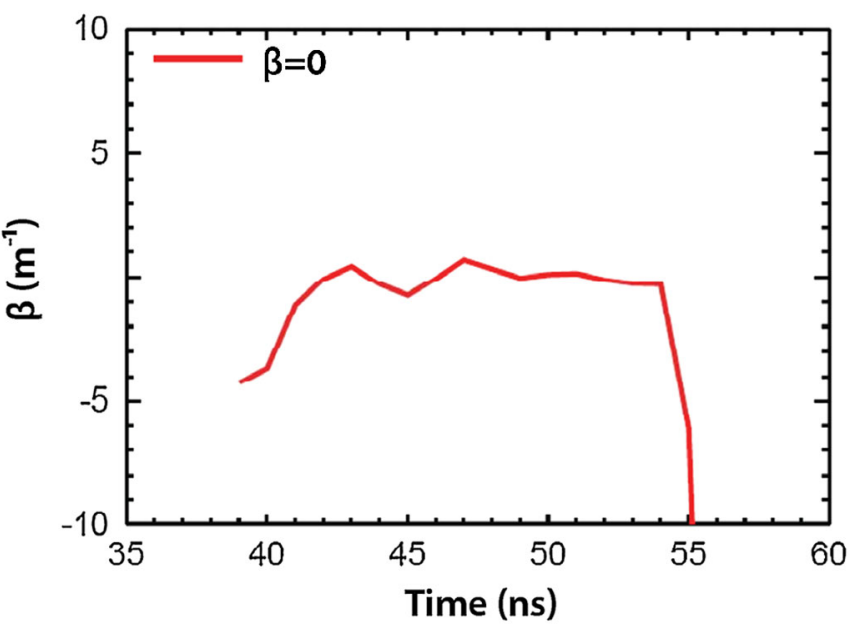

FIG. 7. (Color) Retrapping parameter, $\beta$, versus time for a highvoltage constant electron flow profile. The plateau region of the curve represents the time period of electron flow predominately parallel to the cathode conductor surface. Within the numerical fluctuations, the retrapping rate is essentially constant over the time of interest, although the magnitude of the electron flow is changing significantly.

postulated by the model. The model assumes only that excess electron charge reaches an electrode (either the anode or the cathode). Flow current returning to the cathode (with small electron kinetic energy) is desirable for efficiency and damage reduction reasons. Examination of the charge density within the A-K gap, Fig. 8, reveals that the electron flow becomes more turbulent as the retrapping rate is increased. If the impedance profile is aggressive $\left(\beta \gg 1 \mathrm{~m}^{-1}\right)$, the corresponding buildup of space charge can enable the electron sheath to detach from the cathode and develop into a sheared vortex structure [39,59]. The radial electric field associated with this excess charge can provide vacuum-flowing electrons with the necessary transverse momentum needed to transit the A-K gap and strike the anode [22]. Thus, aggressive retrapping rates can result in electron current to the anode, which is undesirable in most situations. If the transverse current related to this process is capable of initiating an anode surface plasma, the resulting ion emission will limit the reduction in the electron flow current. In high linear current density applications, Ohmic heating of the anode surface becomes the dominant factor in the formation of this anode plasma [33,34].

The mean position of the cathode space charge is illustrated in Fig. 8 and its separation from the anode is associated with the flow impedance of the MITL [21]. For strongly insulated systems, this value is close to the vacuum impedance of the line, resulting from an electron sheath close to the cathode surface. The mean position of the electron sheath extends (from the cathode) further into the A-K gap at radial positions within the vicinity of a vortex. As the retrapping rate increases, these protrusions 

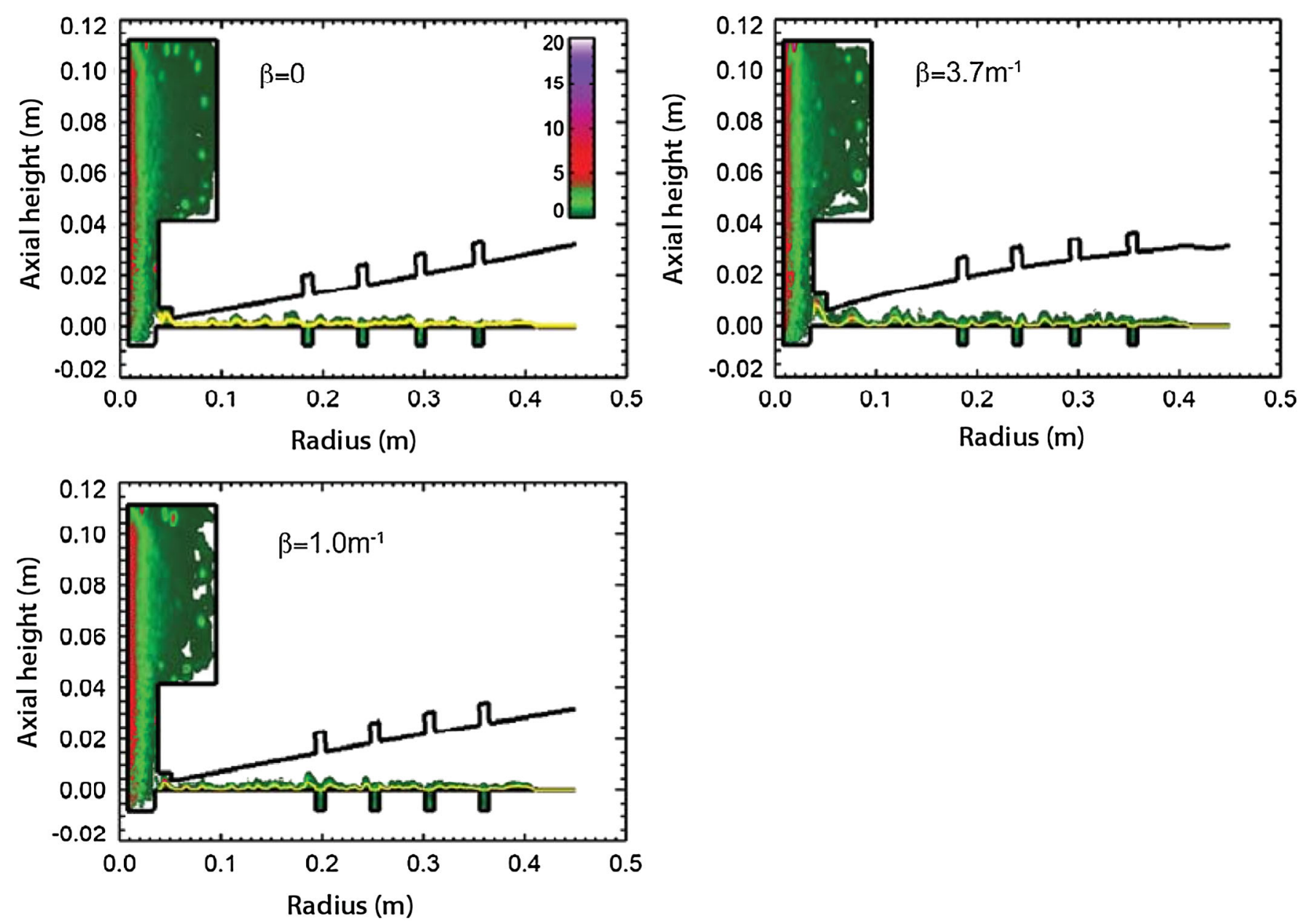

FIG. 8. (Color) Charge density $\left[\mathrm{C} / \mathrm{m}^{3}\right]$ for the $\beta=0,1$, and 3.7 per meter radial profiles at high voltage $(V=5 \mathrm{MV})$. The overlay of the mean position of the cathode space-charge illustrates the flow impedance of the MITL. This line is seen to ripple in the vicinity of electron vortices. As the retrapping rate increases, these vortices become more pronounced near the load.

within the radial profile of the charge centroid become more pronounced near the load.

\section{DISCUSSION}

In this article, an analytic technique for geometrically encoding an impedance profile into the conductors of a strongly insulated MITL, feeding an inductive load, has been developed. For a radial disk configuration, the selfinductance of the transmission line is expressed as a radial function centered at the load, and impedance profiles are derived analytically in terms of the fractional change in the electron flow current with respect to radius. When the vacuum electrons are permitted to return to the cathode conductor (or reach the anode), this fractional change in the flow defines a relative retrapping rate which predicts a radial decrease in the electron flow current in the direction of the power flow. For a desired reduction in electron flow current at the load, these impedance profiles optimize the MITL's self-inductance resulting in only a slight increase in the total vacuum inductance of the system. In many cases, a tailored MITL profile will have lower inductance for a given electron current at its output than would either a constant-impedance or constant-gap MITL profile.

The model presented in Sec. II ignores closure of the MITL's A-K gap and assumes that the local electron flow current operates at values that are consistent with the local application of the 1D pressure-balance model developed in Ref. [15]. PIC calculations, given in Sec. III, confirm that the model is accurate under these assumptions when the disk MITL is operated at sufficiently high voltages, $V>$ $2 \mathrm{MV}$. These simulations establish that the electron flow current at the load can be further reduced through an increase in the analytical model's retrapping rate, $\beta$. As this retrapping rate is increased, however, simulations demonstrate that the corresponding impedance profiles result in the further accumulation of space charge, eventually leading to the formation of electron vortices. These vortices, which facilitate the axial transfer of electrons to the anode surface, are shown to intensify for larger retrapping rates, thus limiting the useful aggressiveness of a reducing flow design. 


\section{ACKNOWLEDGMENTS}

The authors would like to express their appreciation to Dr. Clifford W. Mendel for enlightening discussions regarding this work. Sandia is a multiprogram laboratory operated by Sandia Corporation, a Lockheed Martin Company, for the United States Department of Energy's National Nuclear Security Administration under Contract No. DE-AC04-94AL85000.

[1] R. K. Parker, R. E. Anderson, and C. V. Duncan, J. Appl. Phys. 45, 2463 (1974).

[2] G. A. Mesyats and D. I. Proskurovsky, Pulsed Electrical Discharge in Vacuum (Springer-Verlag, Berlin, 1989).

[3] A. W. Hull, Phys. Rev. 18, 31 (1921).

[4] E. H. Hirsch, Rev. Sci. Instrum. 42, 1371 (1971).

[5] A. Ron, A. A. Mondelli, and N. Rostoker, IEEE Trans. Plasma Sci. 1, 85 (1973).

[6] R. V. Loveless and E. Ott, Phys. Fluids 17, 1263 (1974).

[7] J. M. Creedon, J. Appl. Phys. 46, 2946 (1975).

[8] J. M. Creedon, J. Appl. Phys. 48, 1070 (1977).

[9] K. D. Bergeron, Phys. Fluids 20, 688 (1977).

[10] C. W. Mendel, Jr., J. Appl. Phys. 50, 3830 (1979).

[11] K. D. Bergeron and J. W. Poukey, J. Appl. Phys. 50, 4996 (1979).

[12] C. W. Mendel, Jr., D. B. Seidel, and S. E. Rosenthal, Laser Part. Beams 1, 311 (1983).

[13] C. W. Mendel, Jr., D. B. Seidel, and S. A. Slutz, Phys. Fluids 26, 3628 (1983).

[14] M. S. DiCapua, IEEE Trans. Plasma Sci. 11, 205 (1983).

[15] P. A. Miller and C. W. Mendel, Jr., J. Appl. Phys. 61, 529 (1987).

[16] S. A. Slutz, J. Appl. Phys. 61, 2087 (1987).

[17] R. I. Lawconnell and J. Neri, Phys. Fluids B 2, 629 (1990).

[18] S. E. Rosenthal, IEEE Trans. Plasma Sci. 19, 822 (1991).

[19] C. W. Mendel, Jr., S. E. Rosenthal, and D. B. Seidel, Phys. Rev. A 45, 5854 (1992).

[20] C.W. Mendel, Jr., M.E. Savage, D. M. Zagar, W. W. Simpson, T. W. Grasser, and J.P. Quintenz, J. Appl. Phys. 71, 3731 (1992).

[21] C. W. Mendel, Jr. and S. E. Rosenthal, Phys. Plasmas 2, 1332 (1995).

[22] C. W. Mendel, Jr. and S. E. Rosenthal, Phys. Plasmas 3, 4207 (1996).

[23] C. W. Mendel, Jr. and D. B. Seidel, Phys. Plasmas 6, 4791 (1999).

[24] C. W. Mendel, Jr., J. Appl. Phys. 42, 5483 (1971).

[25] R. A. Vesey, T. D. Pointon, M. E. Cuneo, T. A. Mehlhorn, J. E. Bailey, D. J. Johnson, and W. A. Stygar, Phys. Plasmas 6, 3369 (1999).

[26] T.W. L. Sanford, J.A. Halbleib, J.W. Poukey, A. L. Pregenzer, R. C. Pate, C. E. Heath, R. Mock, G. A. Mastin, and D. C. Ghiglia, J. Appl. Phys. 66, 10 (1989).

[27] M.E. Cuneo, IEEE Trans. Dielectr. Electr. Insul. 6, 469 (1999).

[28] D. W. Swain, S. A. Goldstein, J.G. Kelly, and G. R. Hadley, J. Appl. Phys. 46, 4604 (1975).

[29] A.E. Blaugrund, G. Cooperstein, and S. A. Goldstein, Phys. Fluids 20, 1185 (1977).
[30] L.E. Aranchuk, E. I. Baranchikov, A. V. Gordeev, V. V. Zazhivikhin, V. D. Korolev, and V.P. Smirnov, Zh. Tekh. Fiz. 59, 142 (1989) [Sov. Phys. Tech. Phys. 34, 215 (1989)].

[31] T. D. Pointon, W. A. Stygar, R. B. Spielman, H. C. Ives, and K. W. Struve, Phys. Plasmas 8, 4534 (2001).

[32] P.F. Ottinger and J.W. Schumer, Phys. Plasmas 13, 063101 (2006).

[33] J. W. Schumer, P. F. Ottinger, and C. L. Olson, IEEE Trans. Plasma Sci. 34, 2652 (2006).

[34] P. F. Ottinger and J. W. Schumer, IEEE Trans. Plasma Sci. 35, 154 (2007).

[35] R. B. Spielman, W. A. Stygar, J. F. Seamen, F. Long, H. Ives, R. Garcia, T. Wagoner, K. W. Struve, M. Mostrom, I. Smith, P. Spence, and P. Corcoran, in Proceedings of the 11th IEEE International Pulsed Power Conference, edited by G. Cooperstein and I. Vitkovitsky (IEEE, Piscataway, NJ, 1997), p. 709.

[36] W. A. Stygar, R. B. Spielman, G. O. Allshouse, C. Deeney, D. R. Humphreys, H. C. Ives, F. W. Long, T. H. Martin, M. K. Matzen, D. H. McDaniel, C. W. Mendel, Jr., L. P. Mix, T.J. Nash, J. W. Poukey, J.J. Ramirez, T. W. L. Sanford, J. F. Seamen, D. B. Seidel, J. W. Smith, D. M. Van De Valde, R. W. Wavrik, P. A. Corcoran, J.W. Douglas, I. D. Smith, M. A. Mostrom, K. W. Struve, T. P. Hughes, R. E. Clark, R. W. Shoup, T. C. Wagoner, T. L. Gilliland, and B. Peyton, in Proceedings of the 11th IEEE International Pulsed Power Conference (Ref. [35]), p. 591.

[37] P. A. Corcoran, J. W. Douglas, I. D. Smith, P. W. Spence, W. A. Stygar, K. W. Struve, T. H. Martin, R. B. Spielman, and H.C. Ives, in Proceedings of the 11th IEEE International Pulsed Power Conference (Ref. [35]), p. 466.

[38] E. A. Weinbrecht, D. H. McDaniel, and D. D. Bloomquist, in Proceedings of the 14th IEEE International Pulsed Power Conference, edited by M. Gielsselmann and A. Neuber (IEEE, Piscataway, NJ, 2003), p. 709.

[39] T. A. Hughes, R. E. Clark, B. V. Oliver, T. D. Pointon, and W. A. Stygar, in Proceedings of the 14th IEEE International Pulsed Power Conference (Ref. [38]), p. 622.

[40] T. D. Pointon and M. E. Savage, in Proceedings of the 15th IEEE International Pulsed Power Conference (IEEE, Piscataway, NJ, 2005), p. 151.

[41] K. Hahn, J. Maenchen, S. Cordova, I. Molina, S. Portillo, D. Rovang, D. Rose, B. Oliver, D. Welch, V. Bailey, D. Johnson, and E. Schamiloglu, in Proceedings of the 14th IEEE International Pulsed Power Conference (Ref. [38]), p. 871.

[42] S. Portillo, K. Hahn, J. Maenchen, I. Molina, S. Cordova, D. L. Johnson, D. Rose, B. Oliver, and D. Welch, in Proceedings of the 14th IEEE International Pulsed Power Conference (Ref. [38]), p. 879.

[43] J. Maenchen, G. Cooperstein, J. O'Malley, and I. Smith, Proc. IEEE 92, 1021 (2004).

[44] M.E. Savage, Sandia National Laboratories Internal Memorandum, 2005 (unpublished).

[45] M.E. Savage, J. P. Martin, T. D. Pointon, C. W. Mendel, Jr., D. P. Jackson, W. T. Clark, B. S. Stoltzfus, and M. Pelock, Sandia Laboratories Internal Report, SAND2007-8156, 2008. Copies may be ordered from the National Technical Information Service at www.ntis. gov. 
[46] D. L. Peterson, R. L. Bowers, W. Matuska, and K. D. McLenithan, Phys. Plasmas 6, 2178 (1999).

[47] E. M. Waismann, M.E. Cuneo, W. A. Stygar, R. W. Lemke, K. W. Struve, and T. C. Wagoner, Phys. Plasmas 11, 2009 (2004).

[48] W. A. Stygar, H. C. Ives, D. L. Fehl, M.E. Cuneo, M. G. Mazarakis, J. E. Bailey, G. R. Bennett, D. E. Bliss, G. A. Chandler, R. J. Leeper, M. K. Matzen, D. H. McDaniel, J. S. McGurn, J. L. McKenney, L. P. Mix, D. J. Muron, J. L. Porter, J. J. Ramirez, L. E. Ruggles, J. F. Seamen, W. W. Simpson, C. S. Speas, R. B. Spielman, K. W. Struve, J. A. Torres, R. A. Vesey, T. C. Wagoner, T. L. Gilliland, M. L. Horry, D. O. Jobe, S. E. Lazier, J. A. Mills, T. D. Mulville, J. H. Pyle, T. M. Romero, J. J. Seamen, and R. M. Smelser, Phys. Rev. E 69, 046403 (2004).

[49] C. W. Mendel, Jr., J. A. Swegle, and D. B. Seidel, Phys. Rev. A 32, 1091 (1985).

[50] D. W. Kerst, Phys. Rev. 58, 841 (1940).

[51] D. W. Kerst, Phys. Rev. 60, 47 (1941).

[52] F. R. Elder, R. V. Langmuir, and H. C. Pollock, Phys. Rev. 71, 829 (1947).
[53] F. R. Elder, R. V. Langmuir, and H. C. Pollock, Phys. Rev. 74, 52 (1948).

[54] W. A. Stygar, T. C. Wagoner, H. C. Ives, P. A. Corcoran, M.E. Cuneo, J.W. Douglas, T.L. Gilliland, M.G. Mazarakis, J. J. Ramirez, J. F. Seamen, D. B. Seidel, and R. B. Spielman, Phys. Rev. ST Accel. Beams 9, 090401 (2006).

[55] J.P. Quintenz, D. B. Seidel, M.L. Kiefer, T. D. Pointon, R.S. Coats, S.E. Rosenthal, T.A. Mehlhorn, M.P. Desjarlais, and N.A. Krall, Laser Part. Beams 12, 283 (1994).

[56] C. K. Birdsall and A. B. Langdon, Plasma Physics via Computer Simulation (McGraw-Hill, New York, 1985), Chap. 15.

[57] T. D. Pointon, J. Comput. Phys. 96, 143 (1991).

[58] G. E. Vogtlin and J.E. Vernazza, in Proceedings of the 7th IEEE International Pulsed Power Conference (IEEE Piscataway, NJ, 1989), p. 808.

[59] B. W. Church and R. N. Sudan, Phys. Plasmas 2, 1837 (1995). 